\title{
Analysis of Multilevel-Quantized Soft-Limiting Detector for an FH-SSMA System
}

\author{
Jian F. Weng, Guo Q. Xue, Tho Le-Ngoc, and Sofiène Tahar \\ Dept. of Electrical \& Computer Engineering, Concordia University, Canada \\ email: \{jfweng, xue, tho, tahar\}@ece.concordia.ca
}

\begin{abstract}
In this paper, a multilevel-quantized soft- limiting (SL-MQ) detector for frequency hopping spread spectrum multiple access (FH-SSMA) system is proposed and analyzed. Numerical and simulation results in frequency selective Rayleigh fading channels show that as compared to the hard-limiting (HL) detector, the new SL-MQ with $M=4$ can improve the system capacity by almost $10 \%$ at the bit error rate level of $10^{-3}$. Furthermore, the performance of the SL-MQ has low sensitivity to the optimum value of the amplitude threshold so that it can tolerate inaccurate estimate of its optimum in practice.
\end{abstract}

\section{INTRODUCTION}

Spread spectrum multiple access (SSMA) techniques have attracted considerable attention in personal and mobile communications due to their good anti-jamming capabilities and their potential for high capacity. Of the spread-spectrum signals, the most common forms are direct-sequence (DS) and frequency-hopping (FH). Most studies on spread-

spectrum systems focused on a DS-SSMA because of its potential for higher capacity over FH and the capability of offering diversity reception in a RAKE receiver [1]. However, in DS-SSMA, a stringent synchronization is required inherently, and a very good power-control algorithm is needed in order to minimize the multiple-access interference (MAI) and reduce the "near/far" effect. As an alternative to DSSSMA, FH-SSMA has its own advantages such as nonstringent timing requirement and good immunity to the "nearfar" problem [1]. Various efforts have been devoted to studying the performance and system capacity of FH-SSMA in the presence of partial-band interference, co-channel interference, and fading detriments [2,3]. Recently, for applications of short-range radio-based networks, fast FH is proposed to avoid interference [4]. For an SSMA with fast FH, hard-limiting (HL) detector is commonly used $[2,3]$ and its performance can be shown better than the linear combining detector inasmuch as a serious detriment to the system performance is the non-Gaussian interference incurred by the signals from multiple users $[5,6]$. Such non-Gaussian interference may have an "impulsive" nature (the interference is with a large energy when several users occupy the same frequency slot simultaneously). On the other hand, we also observed that a soft-limiting (SL) detector could provide improved performance over the HL counterpart in suppressing the "impulsive" noise [7]. Therefore, we ex- pect that the SL might outperform the HL in suppressing the non-Gaussian interference in FH-SSMA system.

In this paper, we examine the performance of multilevelquantized soft-limiting (SL-MQ) detector in FH-SSMA system. Here, the quantization is considered for the purpose of implementing a digital detector. Furthermore, as to be shown in the paper, the SL-MQ can reflect the HL [2] or the pure SL by setting the number of levels $(M)$ to 2 or increasing it to $\infty$, respectively. Numerical and simulation results in frequency selective Rayleigh fading channels are presented and they show that the SL-MQ can outperform the HL. In particular, at the bit error rate level of $10^{-3}$, the system capacity can be improved by almost $10 \%$ by using the SL-4Q over the HL (i.e., SL-2Q). The capacity can be further improved by using the SL-MQ with more levels $(M>4)$. Moreover, the performance of the SL-MQ ( $M \geq 4$ ) can be shown to be less sensitive to the optimum value of the threshold $(b)$ as compared to the HL and this means that in practice, the SL-MQ can still work well even with the inaccurate estimate of the optimum value of $b$.

\section{SYSTEM DESCRIPTION}

Consider an FH-SSMA system with MFSK modulation over frequency selective multipath Rayleigh fading channels [2]. A simplified block diagram of an FH-SSMA system can be found in Fig. 1 of [3]. In this system, each user is assigned a unique pseudo-random ( $\mathrm{PN}$ ) address, which is a sequence of $L K$-bit code words. At the transmitter, the $\mathrm{PN}$ address is used to scramble (modulo- $2^{K}$ addition) the buffered $K$-bit message of a user. During the signaling interval $T$, each code word occupies a time slot of duration $\tau=T / L$ and takes on a value between zero and $2^{K}-1$. After passing through the channel, the signal at the receiver is demodulated by means of an MFSK demodulator. The resultant signal is then transformed to the $K$-bit code word and mixed with the address identical to that in the transmitter to remove the scrambled frequency translation. Next, each of the $2^{K}$ frequency slots is determined in a threshold detection manner and thus all detected tones are forming a $2^{K} \times L$ decision matrix. Due to the fact that the detected tones may come from both the desired user and other users in the system, the majority logic decision rule is used, which regards the row having the largest number of entries as the correct one.

We consider $J$ users and assume the first user to be the user of interest and other users as interferers. The PN ad- 
dress for user $u$ is $\bar{A}=\left[a_{0}^{(u)}, a_{1}^{(u)}, \ldots, a_{L-1}^{(u)}\right]$, where $a_{l}^{(u)} \in$ $\left\{0,1, \ldots, 2^{K}-1\right\}$. After dehopping, the output signal at the $t$-th slot and $l$-th tone is given by $[5,8]$

$$
r_{t l}=\delta_{m l} \sqrt{2 \rho^{(0)}} \alpha_{t l}^{(0)}+\sum_{u=1}^{J-1} \gamma_{t l}^{(u)} \sqrt{2 \rho^{(u)}} \alpha_{t l}^{(u)}+z_{t l}
$$

where $m$ is the tone index transmitted by the first user and its presence $(l=m)$ or absence $(l \neq m)$ in the $l$-th tone is indicated by the Kronecker delta $\delta_{m l} .\left\{\rho^{(u)}=E_{\mathrm{W}}^{(u)} /\left(L N_{o}\right)\right.$, $u=0,1, \ldots, J-1\}$ are the normalized signal power per word. $E_{\mathrm{W}}^{(u)}$ is the word energy for user $u$ and $N_{o}$ is the noise spectral density. The sum term in (1) denotes the interference from multiple interferers, in which $\gamma_{t l}^{(u)}$ is an indicating function set to 1 if the $u$-th interferer (after dehopping) occupies the $l$-th tone at the $t$-th time slot and 0 otherwise. $z_{t l}$ is a complex-valued Gaussian noise with zero mean and unit variance and is assumed to be independent and identically distributed (i.i.d.).

In (1), $\left\{\alpha_{t l}^{(u)}, u=0,1, \ldots, J-1\right\}$ for all users are complexvalued fading parameters . We consider Rayleigh fading channel and assume $\alpha_{t l}^{(u)}$ to be Gaussian distributed (the amplitude of $\alpha_{t l}^{(u)}$ is Rayleigh distributed). Here, we restrict $a_{k}^{(u)} \neq a_{l}^{(u)}$ for $k \neq l$ so that the fading parameters are independent from slot to slot [5]. We also assume that (i) fading parameters are independent for different users and (ii) the frequency spacing between the hops is larger than the coherence bandwidth of the Rayleigh fading channel [2, 5]. As a result, the fading parameters $\left\{\alpha_{t l}^{(u)}\right\}$ are mutually independent for all $t, l$, and $u$.

Envelope detection has been commonly used to detect the frequency tone in each time slot as shown in $[2,5]$. The test statistics $\left\{Y_{l}\right\}$ for all frequency tones can be obtained as follows.

$$
\begin{aligned}
& y_{t l}=d\left(\left|r_{t l}\right|\right) \\
& Y_{l}=\sum_{t=0}^{L-1} y_{t l} \quad, \quad l=0,1, \ldots, 2^{K}-1
\end{aligned}
$$

where $d(x)$ is a nonlinear decision function, $\left|r_{t l}\right|$ is the amplitude of $r_{t l}$, and $y_{t l}$ is the functioned amplitude level. Finally, the word decision is made in favor of the row corresponding to the largest sum. In other words, if $Y_{n}$ has the largest sum, the $n$-th tone is determined to be the transmitted tone. If more than one row having the largest sum, we choose each of them with equal probability.

Evidently, if $d(x)=x$, all the signals from $L$ slots are combined linearly. Such linear combining is optimal in the single user system with Gaussian noise. In practice, however, due to the non-Gaussian nature of the interference from multiple users as shown in (1), the hard-limiting (HL) function [2,3], defined as $d_{H L}(x)$, i.e.,

$$
d_{H L}(x)= \begin{cases}1, & \text { if } x>b \\ 0, & \text { otherwise }\end{cases}
$$

where $b$ is the threshold, can be shown to render a better performance than the linear one [5].

Motivated by using a soft-limiting (SL) function to robustly suppress the non-Gaussian noise [7], we examine the performance of multi-level quantized soft-limiting (SLMQ) detector (in order to implement a digital receiver), whose decision function is given by

$$
d_{S L-M Q}(x)= \begin{cases}M-1, & \text { if } x>b \\ i, & \text { if } \frac{i}{M-1} b<x \leq \frac{(i+1)}{M-1} b \\ & i=0,1, \ldots, M-2\end{cases}
$$

where $M$ is the number of levels.

One can readily see that by setting $M=2$, the above function $d_{S L-M Q}(x)$ reduces to the hard-limiting function. On the other hand, the SL-MQ function in (5) can also be expressed as $\frac{1}{M-1} d_{S L-M Q}(x)$ to imitate the pure SL function. If $M \rightarrow \infty$, the normalized $\frac{1}{M-1} d_{S L-M Q}(x)$ would become an SL function, i.e., $d(x)=1$ if $x>b$ and $d(x)=x$ otherwise. Here, we use the expression in (5) because it is evident to express the finite output values in binary bits.

\section{Performance AnAlysis}

We extend Goodman's analysis [2], which was for the hard-limiting function in (4) (or, equivalently, the SL-2Q), to analyze the SL-MQ $(M>2)$ function. We also refine the analysis of [2], which limited the maximum number of hits (more than one user occupying the same frequency tone) to be 1 and assumed no hit on the spurious rows, to study the effects of multiple hits and hits on all rows on the performance.

To yield mathematically tractable analysis, we assume that all users are with the same power, i.e., $\rho^{(0)}=\cdots=\rho^{(J-1)}$ $=\rho$, and all fading parameters $\left\{\alpha_{t l}^{(u)}\right\}$ are i.i.d. Gaussian with zero mean and unit variance.

\section{A: Probability of $y_{t l}=q$}

Let $\Gamma_{t l}=\left[\gamma_{t l}^{(1)}, \gamma_{t l}^{(2)}, \ldots, \gamma_{t l}^{(J-1)}\right]^{T}$ denote the state vector representing the presence or absence of the interferers in the $(t, l)$ position (the $l$-th tone at the $t$-th time slot). Each element in the vector $\Gamma_{t l}$ takes the value from the set $\{0,1\}$ and the space of all possible $2^{J-1}$ state vectors is denoted by $S_{\Gamma}$.

Conditioning on $\Gamma_{t l}=\mathcal{A}(\mathcal{A}$ is a particular state vector in the space $\left.S_{\Gamma}\right), r_{t l}$ in (1) is a zero-mean Gaussian random variable with variance $\sigma_{\mathcal{A}}^{2}$. It can be readily shown that $\sigma_{\mathcal{A}}^{2}=2 \rho(w+1)+1$ for $l=m$ and $2 \rho w+1$ for $l \neq m$, where $w:=W(\mathcal{A})$ is the weight of the vector $\mathcal{A}$, i.e., the number of element " 1 " in $\mathcal{A}$. As a result, the conditional 
probability of $y_{t l}=q$ by using (2) is given by

$$
\operatorname{Pr}\left\{y_{t l}=q \mid \Gamma_{t l}=\mathcal{A}\right\}= \begin{cases}e^{-\frac{(q \beta)^{2}}{2 \sigma_{\mathcal{A}}}} & -e^{-\frac{(q \beta+\beta)^{2}}{2 \sigma_{\mathcal{A}}^{2}}}, \\ e^{-\frac{b^{2}}{2 \sigma_{\mathcal{A}}^{2}}}, & q=0,1, \ldots, M-2\end{cases}
$$

where $\beta:=b /(M-1)$ is the duration for each sub-interval.

The unconditional probability can be obtained by taking expectation of $\operatorname{Pr}\left\{y_{t l}=q \mid \Gamma_{t l}=\mathcal{A}\right\}$ over all $\mathcal{A}$ in the space $S_{\Gamma}$. From (6), the probabilities $\operatorname{Pr}\left\{y_{t l}=q \mid \Gamma_{t l}=\mathcal{A}\right\}$ are equal for various $\Gamma_{t l}=\mathcal{A}$ if they have the equal weight. Accordingly, we have

$\operatorname{Pr}\left\{y_{t l}=q\right\}=\sum_{w=0}^{J-1} \operatorname{Pr}\left\{y_{t l}=q \mid W(\mathcal{A})=w\right\} \operatorname{Pr}\{W(\mathcal{A})=w\}$

where $\operatorname{Pr}\left\{y_{t l}=q \mid W(\mathcal{A})=w\right\}$ denotes the probabilities of $\operatorname{Pr}\left\{y_{t l}=q \mid \Gamma_{t l}=\mathcal{A}\right\}$, in which $\left\{\Gamma_{t l}=\mathcal{A}\right\}$ have the same weight.

Note that the probability of a user sending a tone to the $l$-th frequency is $1 / 2^{K}$. Hence, the probability of $w$ users out of $J-1$ interferers sending tones to that position, i.e., $\operatorname{Pr}\{W(\mathcal{A})=w\}$, can be given by

$$
\operatorname{Pr}\{W(\mathcal{A})=w\}=\left(\begin{array}{c}
J-1 \\
w
\end{array}\right)\left(\frac{1}{2^{K}}\right)^{w}\left(1-\frac{1}{2^{K}}\right)^{J-1-w}
$$

Compared with Goodman's analysis [2], where the maximum number of hits on the spurious rows $(l \neq m)$ was limited to $1, \operatorname{Pr}\left\{y_{t l}=q\right\}$ in (7) considers the number of hits from 0 to $J-1$. Furthermore, [2] did not consider the hits from interferers on the correct row $(l=m)$, where here the hits on all (spurious and correct) rows are considered. The effects of multiple hits and hits on all rows on the performance will be shown later in the next section.

\section{B: Probability of $Y_{l}=i$}

After the combining of $y_{t l}, Y_{l}$ in (3) takes values from 0 to $(M-1) \times L$. For the HL, we can calculate the probability of $Y_{l}=i$ by using the binomial expansion as shown in [2]. For a general SL-MQ and large $L$, however, such calculation requires tremendously computational effort. Hence, we employ the characteristic function $(\mathrm{CF})$ method [9] instead. It can be shown that

$$
\operatorname{Pr}\left\{Y_{l}=i\right\}=\int_{-1 / 2}^{1 / 2}\left[\Phi_{y}(2 \pi f)\right]^{L} e^{-j i 2 \pi f} d f
$$

where $\left[\Phi_{y}(2 \pi f)\right]^{L}$ is the $\mathrm{CF}$ of $Y_{l}$, in which $\Phi_{y}(\omega=2 \pi f)$ is the CF of $y_{t l}\left(\left(\operatorname{Pr}\left\{y_{t l}=q\right\}\right.\right.$ in (7)) given by

$$
\Phi_{y}(\omega)=\sum_{q=0}^{M-1} \operatorname{Pr}\left\{y_{t l}=q\right\} e^{j q \omega}
$$

One can readily check that when $M=2, \operatorname{Pr}\left\{Y_{l}=i\right\}$ in (9) can be easily expressed in a binomial expansion identical to that in [2].

\section{C: Probability of $P(n, k)[2]$}

$P(n, k)$ denotes the probability that $n$ is the maximum number of $Y_{l}$ and that exactly $k$ unwanted (spurious) rows having the value of $n$.

The approach in [2] is adopted to compute $P(n, k)$ after $\operatorname{Pr}\left\{Y_{l}=i\right\}$ evaluated by (9). For notational simplicity, we define $P_{s}(i):=\operatorname{Pr}\left\{Y_{l}=i \mid l \neq m\right\}$ as the probability of $Y_{l}=i$ in the spurious rows $(l \neq m)$ and $P_{c}(i):=\operatorname{Pr}\left\{Y_{l}=\right.$ $i \mid l=m\}$ the probability in the correct row $(l=m)$.

Over $2^{K}-1$ incorrect (spurious) rows, we have

$$
\begin{gathered}
P(n, k)=\left(\begin{array}{c}
2^{K}-1 \\
k
\end{array}\right)\left[P_{s}(n)\right]^{k}\left[\sum_{i=0}^{n-1} P_{s}(i)\right]^{2^{K}-1-k} \\
n=1,2, \ldots,(M-1) L \\
P\left(0,2^{K}-1\right)=\left[P_{s}(0)\right]^{2^{K}-1}, \quad n=0, k=2^{K}-1 \\
P(0, k)=0, \quad n=0, k \neq 2^{K}-1
\end{gathered}
$$

\section{D: Error rate}

The word error rate (WER) is given by [2] but with ( $M-$ 1) $L$ instead of $L$

$$
P_{\mathrm{W}}=1-\sum_{i=0}^{(M-1) L} P_{c}(i) \sum_{k=0}^{2^{K}-1} \frac{1}{k+1} P(i, k)
$$

The bit error rate (BER) is thus given by

$$
P_{b}=\frac{2^{K-1}}{2^{K}-1} P_{\mathrm{W}}
$$

It is noteworthy to mention that the above analysis is not limited to the Rayleigh fading channel but applicable to the additive white Gaussian noise (AWGN) and other kinds of fading channels. For instance, in AWGN channel, we may let $\alpha_{t l}^{(u)}$ to $e^{j \phi_{t l}^{(u)}}$, where $\phi_{t l}^{(u)}$ is the phase angle uniformly distributed over $[0,2 \pi)$, and re-evaluate $\operatorname{Pr}\left\{y_{t l}=q \mid \Gamma_{t l}=\right.$ $\mathcal{A}\}$ in (6), while all other formulas can be kept unchanged to evaluate the BER performance. Further discussion is omitted here for the sake of brevity.

\section{ILlustrative Results}

In this section, several numerical and simulation results are presented to illustrate the performance of the SL-MQ detector. The numerical results are computed by using the formulas in the previous section. The simulation results are obtained by the Monte Carlo trials following the system model in Section 2.

We consider the radio system with the same parameters as those in [2], i.e., $K=8$ and $L=19$ which gives the maximum number of users under the condition of $P_{b} \leq$ $10^{-3}$ in an FH-SSMA system with the bandwidth $=20 \mathrm{MHz}$ and bit rate $=32 \mathrm{~kb} / \mathrm{s}$. Here, a frequency selective Rayleigh 


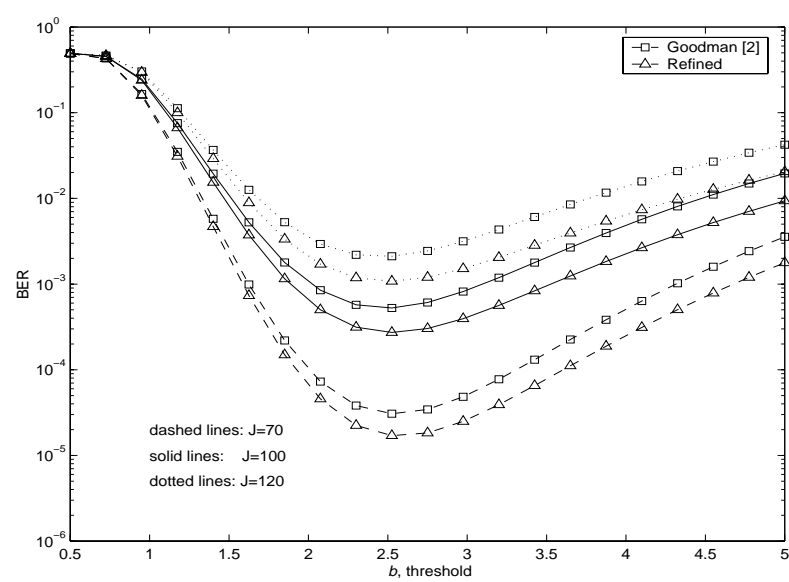

Fig. 1. HL (SL-2Q) BER comparison of Goodman's [2] and our refined analysis in an FH SSMA system with $\mathrm{SNR}=20 \mathrm{~dB}$.

fading channel is considered and the signal-to-noise ratio per bit (SNR) is defined to be $\rho / K$.

A comparison of Goodman's analysis [2] and our refined one evaluated via the formulas in Section 3 on the BERs of the HL (SL-2Q) is shown in Fig. 1 versus the value of $b$ (threshold), where SNR $=20 \mathrm{~dB}$ and $J=70,100,120$. We can see that when $b$ is around 2.5, the HL detector can reach its minimum BER performance. Besides, we note that the BER evaluated by [2] is generally worse than that from (13), though the difference between the corresponding curves is not large. A possible explanation is as follows. Goodman's analysis [2] considered the hits on the spurious row while neglected the hits on the correct row. It is well known that when there are hits on the spurious rows, the system performance will be degraded. However, the hits on the correct row will increase the energy of the signal and result in a little bit better performance than that without considering the hits on the correct row. That is why the BERs computed from [2] are worse than the refined ones.

Next, computer simulation is used to verify the theoretical results. In simulation, we generate a set of random sequences $\{\bar{A}\}$ as the PN addresses for all users and model the received signals according to (1). Each user can select randomly one out of $2^{K}$ available frequency tones to transmit. Suppose the selected tone for user $u$ is $l^{(u)}$. Thus, $\gamma_{t l}^{(u)}$ is set to one if $l^{(u)}+a_{t}^{(u)}-a_{t}^{(0)} \bmod 2^{K}$ equals $l^{(0)}$ and zero otherwise. After obtaining $r_{t l}$, we compute $y_{t l}$ and $Y_{l}$ using (2) and (3), respectively. Next, we make the word decision in favor of the row corresponding to the largest sum or choose one with equal probability of several rows having the largest sum. A counter is used to count the word errors whenever the decision row is different from the transmitted tone. Finally, $3 \times 10^{6}$ trials are carried out to estimate the word error rate (WER). The simulation results on the WER of the HL (SL-2Q) in an FH-SSMA system with $\mathrm{SNR}=20 \mathrm{~dB}, b=2.75$, and $J=70,100,120$ are given in
TABLE I

SiMULATION AND THEORETICAL WER's OF THE HL (SL-2Q) IN AN FH-SSMA WITH SNR=20DB.

\begin{tabular}{|l|l|l|l|}
\hline \# of users & Goodman [2] & Refined & Simulation \\
\hline 70 & $6.85 \mathrm{e}-5$ & $3.65 \mathrm{e}-5$ & $4.73 \mathrm{e}-5$ \\
\hline 100 & $1.21 \mathrm{e}-3$ & $6.03 \mathrm{e}-4$ & $6.65 \mathrm{e}-4$ \\
\hline 120 & $4.86 \mathrm{e}-3$ & $2.39 \mathrm{e}-3$ & $2.47 \mathrm{e}-3$ \\
\hline
\end{tabular}

Table 1 in comparison with the theoretical ones computed via Goodman's analysis [2] and our refined one (derivation in Section 3). It can be seen that the simulation results on the WERs are close to the refined ones evaluated via (12), but deviated from those by [2]. This confirms our analysis.

Noting that the derivation in Section 3 considered more than one hit while [2] limits the maximum number of hits on a spurious row to be 1 , one may be interested to know the effect of the number of hits on the performance. To study this, we assume $N_{h}$ to be the maximum number of hits being limited, and define

$\operatorname{Pr}\left\{W\left(\mathcal{A}^{\prime}\right)=w\right\}= \begin{cases}\operatorname{Pr}\{W(\mathcal{A})=w\}, & w \leq N_{h} \\ \sum_{i=N_{h}}^{J-1} \operatorname{Pr}\{W(\mathcal{A})=i\}, & w=N_{h}\end{cases}$

where $\mathcal{A}^{\prime}$ is the state vector in $S_{\Gamma}$ but its weight is no larger than $N_{h}$ and $\operatorname{Pr}\{W(\mathcal{A})=w\}$ is evaluated from (8).

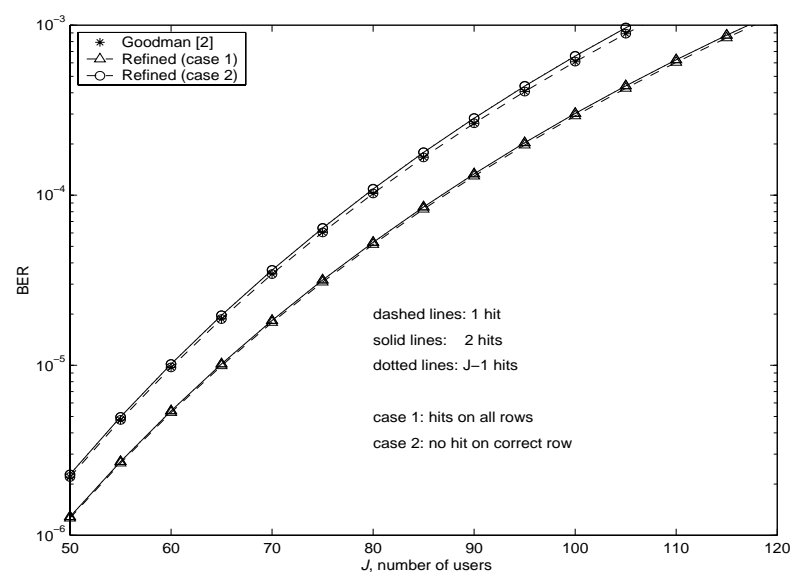

Fig. 2. Effect of the maximum number of hits on the BER of the HL(SL$2 \mathrm{Q}$ ) in an FH SSMA system with SNR=20dB.

Now, we evaluate the BER as a function of $N_{h}$ following the procedures in Section 3 but using $\operatorname{Pr}\left\{W\left(\mathcal{A}^{\prime}\right)=w\right\}$ in (14) instead of (8). To compare with Goodman's method [2], where the hit on the correct row was not considered, we proceed our evaluation into two cases. In case 1 , we consider hits on all rows, while in case 2, we limit hits on the spurious rows but no hit on the correct row (this is the worst case as previously mentioned). The resulting BERs of the HL (SL-2Q) versus the number of users are plotted 
in Fig. 2, where $N_{h}=1,2, J-1$. The results, once again, show that by considering the hits on the correct row (case 1 ), the performance is better than that with no hits on the correct row (case 2). Also, as predicted, the curve with that considering 1 hit in spurious rows (case 2, $N_{h}=1$ ) is in agreement with that from Goodman's analysis [2]. Furthermore, by considering more hits ( 2 or $J-1$ hits), the performance (the corresponding two curves are overlapping) is becoming worse. However, the performance degradation in comparison with that for $N_{h}=1$ is almost negligible due to the fact that there is a large number of available frequency tones $\left(2^{K}=256\right)$ and the probability of many hits is very small. This suggests that for practical evaluation, we can limit the maximum number of hits to one.

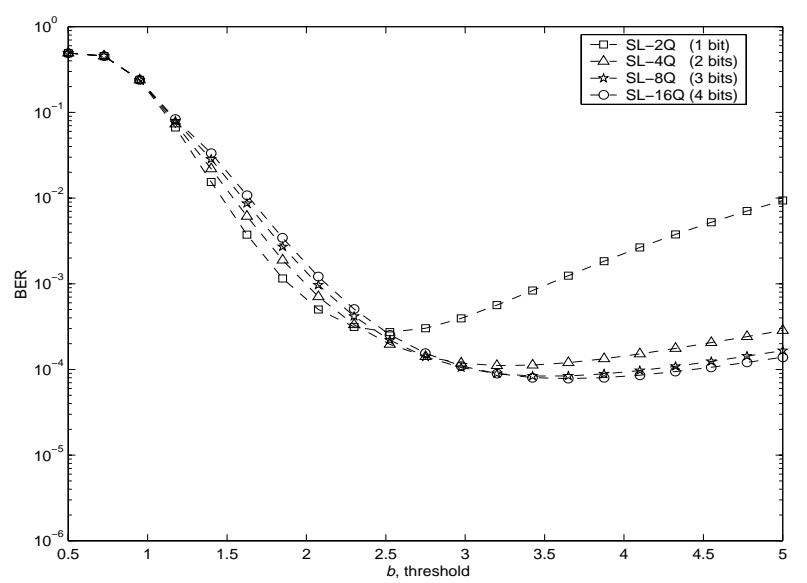

Fig. 3. Effect of the threshold (b) on the BERs of the SL-MQ detector $\mathrm{SNR}=20 \mathrm{~dB}$.

Next, we delve the performance of the SL-MQ detector for more levels $(M>2)$. In Fig. 3, we compare the BERs of the SL-2Q (1 bit), SL-4Q (2 bits), SL-8Q (3 bits), and SL-16Q (4 bits) in an FH-SSMA system with SNR=20dB and $J=100$ users. Here, $1,2,3,4$ bits denote the required numbers of bits used to express $M=2,4,8,16$ levels, respectively. All BERs are evaluated from Section 3 . The SL$2 \mathrm{Q}$ is the HL detector as considered in $[2,3,5]$. The figure clearly indicates that, for the SL-4,8,16Q, the corresponding optimum values of $b$ for threshold become larger as compared with the HL (SL-2Q). Besides, the performance of the SL-MQ $(M>2)$ is shown less sensitive to the optimum value of the threshold $b$ than that of the SL-2Q. This property is very useful in practice when it is difficult to estimate the optimum $b$ accurately.

To further study the performance of the SL-MQ, Fig. 4 plots the BERs of the SL-MQ $(M=2,4,8,16)$ versus the number of users $(J)$ at $\mathrm{SNR}=20 \mathrm{~dB}$. The optimum values of $b$ for the SL-2,4,8,16Q are set to $2.5,3.25,3.5$ and 3.75, respectively, which are roughly estimated from the previous figure. From Fig. 4, we can see that compared with the SL$2 \mathrm{Q}$, the SL-MQ $(\mathrm{M}=4,8,16)$ can improve the system capacity. For example, at the BER level of $10^{-3}$, the SL-4Q can improve the system capacity by almost $10 \%$, i.e., from 118 users to 130 users. The penalty paid for this is the increase in the hardware complexity. For the SL-4Q ( 2 bits), the memory required is doubled as compared to that for the SL2Q. With the SL-8Q or SL-16Q, the capacity improvement is even larger and the memory required would be tripled or four times.

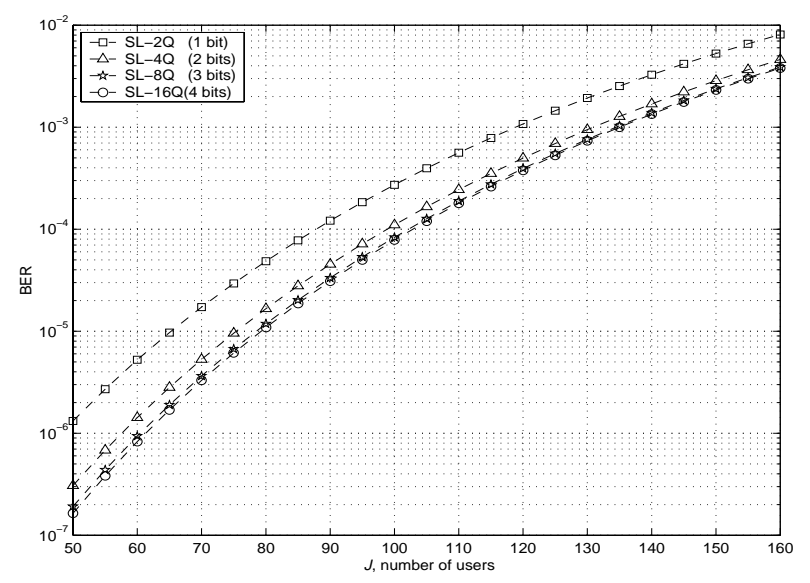

Fig. 4. BERs of the SL-MQ detector versus the number of users $(J)$. $\mathrm{SNR}=20 \mathrm{~dB}$.

\section{ACKNOWLEDGMENT}

This work is partially supported by Ericsson Research Canada.

\section{REFERENCES}

[1] C. D'Amours and A. Yongacoglu, "MFSK based modulations for hybrid DS/FH CDMA systems operating in slowly Rayleigh fading channels," IEEE Trans. Vehi. Tech., vol. VT-46, pp. 615-624, Aug. 1997.

[2] D. J. Goodman, P. S. Henry, and V. K. Prabhu, "Frequency hopped multilevel FSK for mobile radio," Bell System Technical J., pp. 12571275, Sept. 1980.

[3] U. Svasti-Xuto, Q. Wang, and V. K. Bhargava, "Capacity of an FHSSMA system in different fading environments," IEEE Trans. Vehi. Tech., vol. VT-47, pp. 75-82, Feb. 1998.

[4] Bluetooth, "Bluetooth system," in http://www.bluetooth.com, Special Interest Group (SIG), 1999.

[5] O. C. Yue, "Performance of frequency hopping multiple access multilevel FSK systems with hard limited and linear combining," IEEE Trans. Commun., vol. COM-29, pp. 1687-1694, Nov. 1981.

[6] J. Ilow, D. Hatzinakos, and A. N. Venetsanopoulos, "Performance of FH SS radio networks with interference modeled as a mixture of Gaussian and Alpha-Stable noise," IEEE Trans. Commun., vol. COM-46, pp. 509-519, April 1998.

[7] J. F. Weng, Performance of two-stage nonlinear detector in DS/CDMA system with impulse noise. $\mathrm{PhD}$ thesis, Dept. of Electronic Eng., City Univ. of Hong Kong, Hong Kong, Aug. 1997.

[8] T. Y. Yan and C. C. Wang, "Mathematical models for cochannel interference in FH/MFSK multiple access systems," IEEE Trans. Commun., vol. COM-32, pp. 670-678, June 1984.

[9] A. Papoulis, Probability, random variables, and stochastic processes. New York: McGraw-Hill, 1991. 